\title{
Melhor prevenir do que remediar: Avaliando usabilidade e UX de software antes de levá-lo para a sala de aula
}

\author{
Ingrid Nascimento ${ }^{1}$, Wagner Gaspar ${ }^{2}$, Tayana Conte ${ }^{1}$, Bruno Gadelha ${ }^{1}$, Elaine H. \\ T. Oliveira ${ }^{1}$ \\ ${ }^{1}$ Instituto de Computação - Universidade Federal do Amazonas (UFAM) \\ CEP 69.077-000 - Manaus - AM - Brasil \\ ${ }^{2}$ Departamento de Informática - Universidade Federal do Espírito Santo (UFES) \\ CEP 29.075-910 - Vitória - ES - Brasil \\ \{inc,wg, tayana, bruno, elaine\} @icomp.ufam.edu.br
}

\begin{abstract}
Technological tools for education may emerge from academic environments. However, it is important to evaluate their quality before adopting them in school context. In this work, an application that explores the origin of Portuguese words was developed. Usability and UX (User eXperience) evaluation was held to verify application suitability and ease of use interaction. To the surprise of the developers, several problems were found and suggestions for improvements were made. Thus, the main theme of this work is to encourage this type of development, taking into account the importance of submitting educational software for usability and UX inspections to prevent major problems.
\end{abstract}

Resumo. Ferramentas tecnológicas aplicadas à educação podem surgir de ambientes acadêmicos. No entanto, antes de adotá-las no contexto escolar é importante avaliar sua qualidade. Este trabalho apresenta um aplicativo que explora a origem das palavras da língua portuguesa. Para verificar a adequação do aplicativo e a facilidade de interação dos usuários foi realizada uma avaliação de Usabilidade e UX (User eXperience). Para surpresa dos desenvolvedores, foram detectados vários problemas e melhorias foram sugeridas. Assim, o mote principal deste trabalho é incentivar esse tipo de desenvolvimento, levando em consideração a importância de submeter esse tipo de software a inspeções de usabilidade e UX como medida preventiva.

\section{Introdução}

Universidades e outras instituições de ensino são espaços propícios para iniciativas de pesquisa, desenvolvimento e inovação. Em ambientes dinâmicos, em que há interação entre pesquisadores e alunos em formação, tanto em nível de graduação quanto de pósgraduação, é natural que o conhecimento construído acabe se transformando em produtos inovadores.

Nesse contexto, a educação tem sido beneficiada com a proposta de utilização de ferramentas tecnológicas que apoiem o processo de ensino-aprendizagem. A gama de pesquisas na área de Informática na Educação é ampla e diversificada, atendendo a várias questões de investigação. $\mathrm{Na}$ área de ensino da língua portuguesa, um dos temas 
ainda pouco atendidos e que, muitas vezes, causa confusão entre os alunos é a grafia correta das palavras. Nota-se também que há poucos aplicativos que contemplam tal tema [Gaspar, Oliveira e Oliveira 2015]. Na tentativa de atender tal demanda, foi iniciado o processo de desenvolvimento de um aplicativo que explorasse o assunto, baseado na origem das palavras. Ao final do processo de desenvolvimento, foi conduzida uma avaliação de Usabilidade e UX (User eXperience) da primeira versão do aplicativo. Para surpresa dos desenvolvedores do aplicativo, foram detectados vários problemas, a maioria relatada neste artigo. A partir desse resultado, surgiu a motivação para este relato, cujo mote principal é incentivar a pesquisa e o desenvolvimento em ambientes acadêmicos, mas sempre levando em consideração a importância de submeter os aplicativos e softwares a avaliações de usabilidade e UX antes de levá-los à sala de aula.

A usabilidade é um dos atributos de qualidade mais importante e, segundo a norma ISO/IEC 25010 (2011), é definida como a "capacidade do produto de software de ser compreendido, aprendido, operado e atraente ao usuário, quando usado sob condições específicas". De la Vara et al. (2011) realizaram uma pesquisa sobre a importância relativa de cada um dos atributos de qualidade de software. Os resultados desta pesquisa revelaram que a maioria dos participantes considerou a usabilidade como o atributo mais importante. Deste modo, diversos métodos de avaliação têm sido propostos com o objetivo de identificar problemas de usabilidade das aplicações interativas, aumentando a facilidade de interação dos usuários ao utilizarem tais aplicações.

No caso de aplicativos para apoio ao ensino, é importante considerar a usabilidade sob duas perspectivas: a necessidade de considerar tanto a usabilidade de aplicações móveis quanto a usabilidade de softwares educacionais. Com o crescente uso de smartphones e tablets, o número de aplicativos e jogos para dispositivos móveis tem aumentado também e com isso, surgem novas propostas de técnicas de avaliação talvez mais adequadas ao processo de desenvolvimento rápido e peculiar [Bonifácio et al. 2010, Kawakami et al. 2014]. Outra preocupação é que, em softwares educacionais, problemas de usabilidade podem não apenas dificultar o uso do sistema como prejudicar a aprendizagem dos conteúdos apresentados [Reategui 2007].

Todo o processo e reflexões advindas da experiência serão relatados através da seguinte estrutura: a Seção 2 apresenta trabalhos que também consideram a importância de se avaliar a usabilidade e a UX em aplicações móveis e/ou educacionais. Na Seção 3, o processo de desenvolvimento do aplicativo é descrito. Alguns pontos importantes sobre usabilidade e UX são apresentados na Seção 4, com ênfase na técnica utilizada, a Userbility. O resultado da avaliação do aplicativo e a discussão sobre sua importância estão na Seção 5. Finalmente, a Seção 6 apresenta as considerações finais e os trabalhos futuros.

\section{Usabilidade e UX em softwares educacionais}

Visando identificar um método com bom desempenho na avaliação de usabilidade de aplicações Web móveis, Bonifácio et al. (2010) apresentam um estudo comparativo entre os métodos: Avaliação Heurística (AH), Percurso Cognitivo (PC) e Web Design Perspectives-based Usability Evaluation (WDP). Segundo os autores, o WDP encontrou 
V Congresso Brasileiro de Informática na Educação (CBIE 2016)

Anais do XXVII Simpósio Brasileiro de Informática na Educação (SBIE 2016)

o maior número de problemas, podendo ter um desempenho satisfatório na avaliação de aplicações Web móveis, o PC pode ser adaptado facilmente para o contexto móvel enquanto que o $\mathrm{AH}$, pode ser utilizada em vários padrões de aplicações, sendo a mais adequada tendo em vista os resultados obtidos e o tempo gasto para a aplicação da técnica. Ainda no contexto de aplicações Web móveis, Kawakami et al. (2014) apresentam um conjunto de tecnologias de inspeção de usabilidade a serem aplicadas ainda no processo de design, diminuindo assim o custo de correção de problemas antes mesmo da escrita do código fonte da aplicação.

Em Penedo et al. (2012), é proposta a análise de usabilidade de sistemas Web usando inspeção baseada em modelos de Markov em conjunto com inspeção baseada em taxonomia, sendo apresentado um estudo de caso de um sistema de ensino a distância a fim de verificar a viabilidade da proposta. Os autores destacam ainda que os métodos de avaliação diferem entre si em vários aspectos, sendo fundamental compreender essas diferenças e assim poder definir o mais apropriado para o objetivo desejado, nesse caso, a aprendizagem.

As preocupações com a usabilidade em softwares educacionais são várias e estão relacionadas com a influência desses softwares na aprendizagem. Catapan et al. (1999) consideram a possível integração entre usabilidade e aprendizagem através do que chamam de ergonomia em software educacional. Chegaram à conclusão que, para uma avaliação mais abrangente, seria necessário: "uma equipe multidisciplinar, tempo e condições logística suficientes; uma amostra significativa de usuários, e exploração da totalidade dos fatores e critérios que compõem o pressuposto de aprendizagem”. Já Atayde et al. (2003) propuseram uma metodologia de avaliação de qualidade de software educacional infantil, a MAQSEI. Apesar de o foco ser no ensino infantil, as heurísticas desenvolvidas colaboram como referência técnica e pedagógica tanto para avaliadores quanto para desenvolvedores. Gomes e Padovani (2005) dizem que "soluções educativas geradas em centros de pesquisa tendem a abordar o problema do desenvolvimento de aplicações educativas de forma não sistemática", o que pode afetar a qualidade do produto. Reategui (2007) faz uma reflexão sobre a importância da interface para softwares educativos, decompondo a interface em oito aspectos, dentre eles: a interatividade, a usabilidade e a afetividade. Sua premissa é que, assim como um livro bem diagramado e pensado, uma boa interface influencia a aprendizagem.

Esses temas devem nortear o restante do artigo e auxiliar nas considerações finais e nas sugestões de trabalhos futuros. A seguir, o processo de desenvolvimento do aplicativo é descrito para melhor entendimento do caso a ser considerado.

\section{O aplicativo NaPontaDaLíngua}

O desenvolvimento do aplicativo foi baseado na metodologia ágil SCRUM, com ênfase em entregas iterativas e incrementais. Inicialmente, um protótipo foi criado na ferramenta Pencil versão 2.0.5. Esta permite não apenas a criação das telas, mas também a criação de links entre elas, permitindo assim a simulação do funcionamento do aplicativo.

O aplicativo foi projetado de tal forma que o estudante pudesse utilizar boa parte dos recursos sem estar conectado à Internet. Todas as informações referentes à LP ficam armazenadas no SQLite, o Sistema Gerenciador de Banco de Dados do Android 
V Congresso Brasileiro de Informática na Educação (CBIE 2016)

Anais do XXVII Simpósio Brasileiro de Informática na Educação (SBIE 2016)

(SGBD), não interferindo em sua utilização caso não esteja conectado à Internet. Algumas opções permitem adicionar comentários ou dúvidas, neste caso se faz necessário estar conectado à Internet e criar uma conta ou acessar usando uma conta do Facebook. Essas informações ficam armazenadas em um servidor Web com o SGBD MySql criado para essa finalidade. No processo de desenvolvimento foi escolhida a plataforma Android em sua versão 4.0 por ser aberta e amplamente utilizada, fazendo uso da IDE Android Studio versão 1.0.

Na Figura 1a é apresentada a tela inicial do aplicativo, onde o estudante pode escolher entre treinar suas habilidades, acessar o dicionário, o modo Grafo ou o modo Jogo. Se o modo treino for escolhido, a tela da Figura 1 b será exibida e nela são apresentadas quatro opções de grafia para a mesma palavra, devendo o estudante clicar sobre a opção que julgar correta. Feito isso, ele será direcionado para a tela apresentada na Figura 1c, indicando se ele acertou ou não, apresentando a escrita correta da palavra e uma breve descrição de sua origem. Nessa tela, o estudante ainda pode postar uma dúvida ou comentário ou mesmo informar um erro (opções que dependem de acesso à Internet).

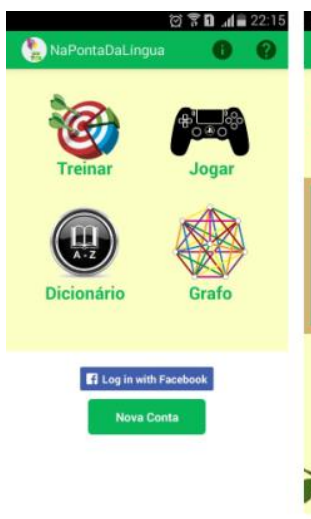

(a)

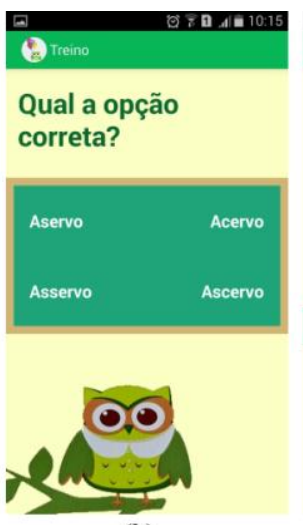

(b)

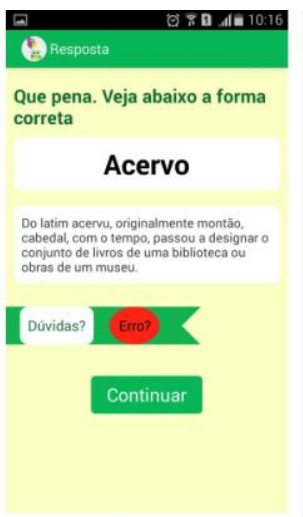

(c)

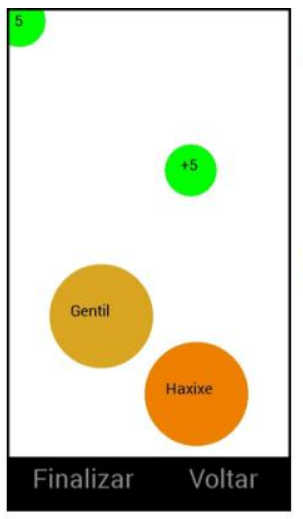

(d)

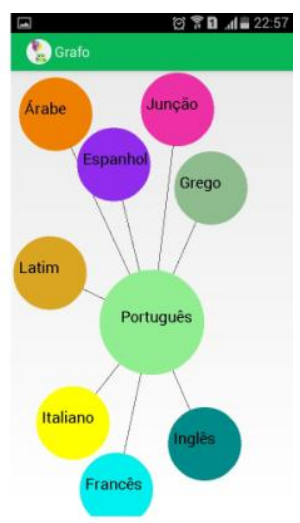

(e)

Figura 1. Telas do aplicativo

Ao escolher a opção Dicionário, será exibida uma lista com todas as palavras cadastradas na base de dados do aplicativo (50 até o momento), bastando clicar sobre uma delas para visualizar suas informações. No modo Jogo, apresentado na Figura 1d, o estudante deve estourar os balões cujas palavras estejam grafadas corretamente, e ao final, é apresentada uma lista com a pontuação e as palavras que ele errou durante o jogo. Por fim, ao acessar o modo Grafo, o estudante visualizará a tela da Figura 1e, onde são apresentadas todas as línguas (cadastradas no aplicativo) que contribuíram para a formação da LP. Caso deseje consultar as palavras de uma língua específica (do grego, por exemplo), basta clicar sobre o balão da língua grega e todas as palavras provenientes desta língua (cadastradas no aplicativo) serão apresentadas. Caso queira visualizar as informações de uma palavra, basta clicar sobre a mesma.

\section{Avaliação de usabilidade e UX}

Os Métodos de Avaliação de Usabilidade (Usability Evaluation Methods - UEMs) são procedimentos compostos por um conjunto bem definido de atividades que são usadas 
V Congresso Brasileiro de Informática na Educação (CBIE 2016)

Anais do XXVII Simpósio Brasileiro de Informática na Educação (SBIE 2016)

para avaliar a usabilidade de uma aplicação [Fernandez et al. 2011]. Os métodos comumente adotados para avaliação de usabilidade podem ser divididos em duas categorias: (1) Inspeções de Usabilidade, nas quais inspetores examinam aspectos da aplicação para detectar violações de princípios de usabilidade estabelecidos; e (2) Testes de Usabilidade, que são métodos de avaliação baseados na participação direta de usuários. Embora o teste de usabilidade seja considerado o método mais eficaz para avaliar sistemas e protótipos do ponto de vista do usuário das aplicações, seu custo é alto, pois envolve o tempo dos usuários e muitas vezes o uso de laboratórios específicos de usabilidade [Matera et al. 2002]. Os métodos de inspeção foram propostos como uma alternativa com bom custo-benefício em comparação aos testes de usabilidade.

A usabilidade é considerada parte da experiência do usuário. A experiência do usuário considera também a satisfação ao realizar uma determinada tarefa, além de fatores físicos, ambientais e emocionais inerentes ao contexto de utilização do sistema. A avaliação de UX implica na ampliação dos métodos existentes para avaliação de usabilidade [Vermeeren et al. 2010]. Novos métodos de avaliação de UX tem sido propostos, focando principalmente em experiências e análise de emoções de pessoas interagindo com produtos de software [Vermeeren et al. 2010]. No entanto, na indústria, as avaliações de UX e usabilidade têm sido comumente realizadas em separado, sendo algumas técnicas empregadas somente para avaliar usabilidade, enquanto outras verificam os aspectos de UX. Com o objetivo de realizar uma avaliação mais completa, buscou-se empregar técnicas que integram as avaliações de UX e usabilidade em aplicações móveis.

\subsection{Userbility}

A Userbility (Integração de User eXperience e Usability) [Nascimento et al. 2016] também é fruto do ambiente acadêmico e tem como objetivo integrar a avaliação de usabilidade com a avaliação de UX para auxiliar profissionais não especialistas em IHC (Interação Humano-Computador). Essa técnica contém: (1) doze heurísticas de usabilidade para aplicações móveis com (2) itens de verificação, (3-4) duas questões de UX com exemplos para cada heurística de usabilidade e (5) um item relacionado à satisfação dos avaliadores, representado por um rosto humano e uma descrição. A Figura 2, a seguir, mostra um trecho da Userbility para a heurística de usabilidade A1 (Visibilidade do estado do sistema).

Para realização de um estudo experimental de UX e usabilidade para avaliar a aplicação NaPontaDaLíngua, a técnica Userbility foi comparada com um método existente, UX and Usability Guidelines Approach - UUGA [De Paula et al. 2014].

\section{Metodologia de avaliação do NaPontaDaLíngua}

Esta seção apresenta os dois objetivos principais da avaliação: avaliar o aplicativo NaPontaDaLíngua antes de seu uso em sala de aula e validar a técnica Userbility comparando-a com um método existente, o UUGA. Para isso, foi realizado um estudo experimental de avaliação de UX e usabilidade, utilizando essas duas técnicas. A seguir são apresentadas as etapas do estudo experimental. 
V Congresso Brasileiro de Informática na Educação (CBIE 2016)

Anais do XXVII Simpósio Brasileiro de Informática na Educação (SBIE 2016)

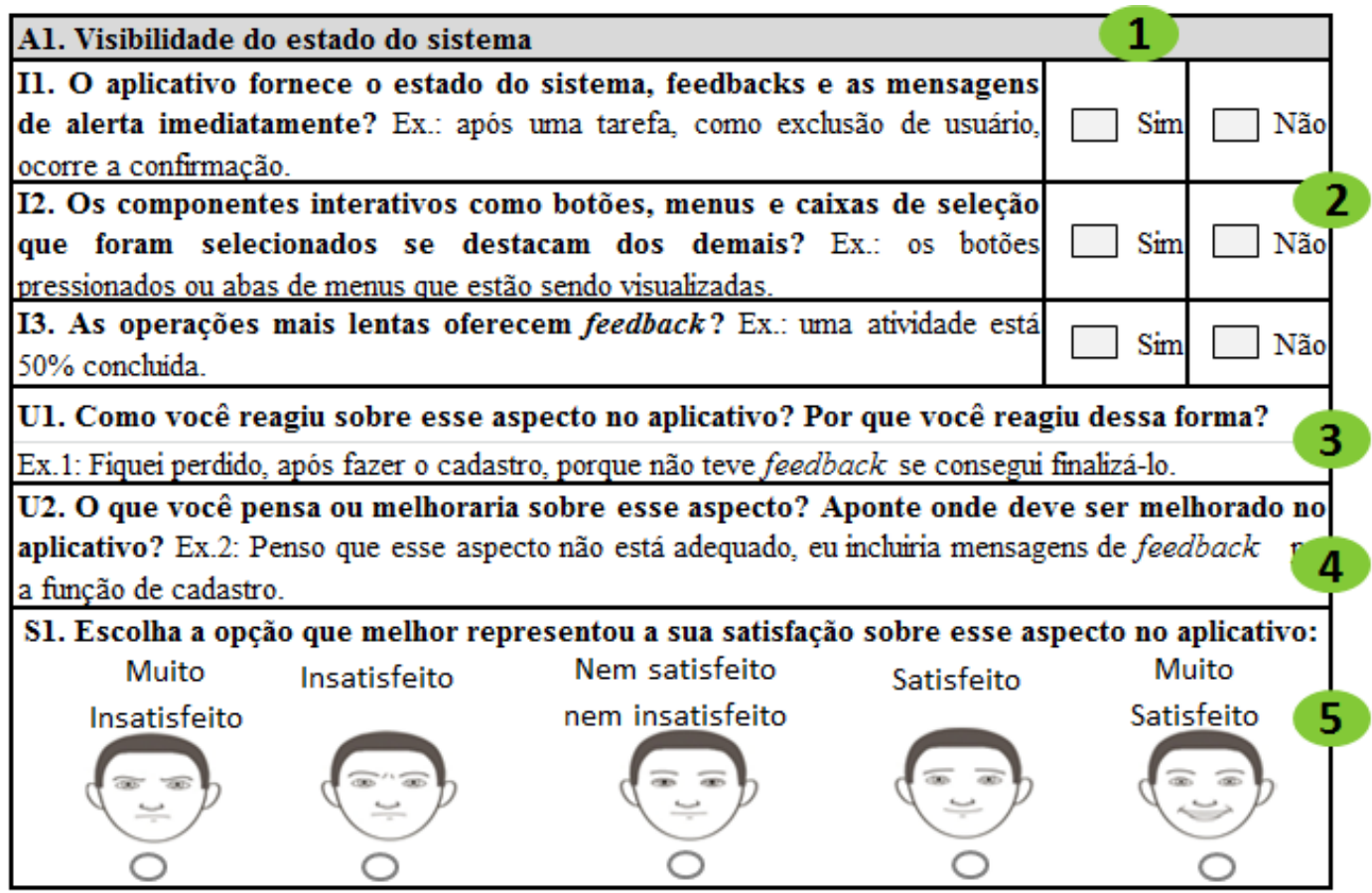

Figura 2. Exemplo de parte da técnica Userbility v 2.0.

\subsection{Planejamento}

Durante a fase de planejamento do estudo da aplicação, foram definidos os seguintes recursos: contexto e participantes, treinamento e definição de tarefas.

Contexto e participantes: foi introduzido o contexto para a aplicação "NaPontaDaLíngua" - "Esta é uma aplicação que visa auxiliar os alunos quando estão aprendendo a língua portuguesa, descrevendo a origem das palavras e ajudando os usuários a corrigir a sua grafia através de um jogo simples”. Os participantes foram 49 estudantes das disciplinas de IHC e Sistemas Colaborativos da Universidade Federal do Amazonas que concordaram em participar do estudo como avaliadores da aplicação. Todos os participantes assinaram um termo de consentimento para participar do estudo e preencheram um questionário de caracterização para medir seus conhecimentos em IHC e seu conhecimento em análise e projeto de software.

Treinamento: foi realizado um treinamento dos participantes que introduziu os conceitos e exemplos de UX e usabilidade ao avaliar um aplicativo. Os participantes foram divididos em dois grupos, onde um grupo recebeu treinamento sobre a Userbility (25 participantes) e o outro sobre a técnica UUGA (24 participantes). Além disso, o grupo da UUGA tinha mais de um participante com um grau elevado de conhecimento em análise e projeto de software quando comparado com o grupo da Userbility.

Definição de tarefas: cada participante realizou seis tarefas principais da aplicação NaPontaDaLíngua. As tarefas foram as seguintes: (a) treinar, respondendo, pelo menos, três questões; (b) iniciar um jogo; (c) visualizar o resultado final do jogo; (d) procurar uma palavra no dicionário; (e) visualizar o gráfico; (f) e encontrar informações sobre dúvidas. 
V Congresso Brasileiro de Informática na Educação (CBIE 2016)

Anais do XXVII Simpósio Brasileiro de Informática na Educação (SBIE 2016)

\subsection{Execução}

O processo de inspeção foi conduzido aplicando as duas técnicas para avaliar a aplicação NaPontaDaLíngua. Os participantes receberam tablets ou celulares para executar as tarefas descritas anteriormente na aplicação utilizando as técnicas propostas. Após cada participante realizar sua avaliação, foram agrupadas as discrepâncias em uma lista, onde foi realizada uma análise para retirar as discrepâncias repetidas. Então foi realizada uma reunião para classificar as discrepâncias em: problemas ou falso positivos (discrepâncias que não foram consideradas problemas que afetam a usabilidade e a UX da aplicação NaPontaDaLíngua). Nessa reunião participaram cinco pesquisadores: um dos criadores da aplicação, um especialista em UX e usabilidade e outros três pesquisadores com bom conhecimento de inspeção, que atuaram como moderadores.

\section{Resultados}

Após a execução da inspeção, foram analisados dados quantitativos e qualitativos. Os quantitativos foram obtidos da lista das discrepâncias resultante da reunião de discriminação, e os qualitativos, das percepções sobre melhorias sugeridas pelos participantes através das técnicas Userbility e UUGA.

A Tabela 1 mostra os resultados por técnica em termos de número de defeitos identificados, defeitos exclusivos e melhorias sugeridas. A análise dos resultados mostra que o UUGA e a Userbility permitiram encontrar quase o mesmo número de defeitos (114 e 116, respectivamente). No entanto, entre os verdadeiros problemas identificados, a Userbility permitiu identificar mais defeitos únicos (total de 37 defeitos únicos) do que o UUGA (total de 31 defeitos únicos), sem contar as duplicatas que foram apontadas por mais de um participante.

Tabela 1. Análise das Discrepâncias por técnica

\begin{tabular}{|c|c|c|c|}
\hline Técnica & $\begin{array}{c}\text { Total de } \\
\text { Defeitos }\end{array}$ & $\begin{array}{c}\text { Defeitos } \\
\text { Únicos }\end{array}$ & $\begin{array}{c}\text { Sugestões } \\
\text { de Melhoria }\end{array}$ \\
\hline Userbility & 116 & 37 & 190 \\
\hline UUGA & 114 & 31 & 11 \\
\hline
\end{tabular}

Além disso, o número de melhorias sugeridas ao utilizar a Userbility (190) foi bem mais elevado do que o número de melhoramentos sugeridos ao usar o UUGA (11). As melhorias sugeridas pelos participantes foram relacionadas com os problemas identificados na aplicação. A seguir, algumas das melhorias sugeridas para alguns problemas são apresentadas:

Muito esforço para realizar tarefas simples - "Deveria ser adicionado [sic] uma barra de busca na tela de dúvidas."; "Acho que poderia haver uma revisão sobre a execução de algumas tarefas.".

Erros na aplicação - "Deveria ter uma mensagem do ocorrido ou um log para os desenvolvedores resolverem.”; "Uma opção de tirar os balões que estão bloqueando outros no modo jogar.".

Dificuldade de entendimento - "Mudaria os nomes das telas, pois estão confusos."; "Acho que poderia adicionar um botão de ajuda sobre as funcionalidades do app."; "O texto é curto, deveria ser algo como: Achou algum erro?". 
V Congresso Brasileiro de Informática na Educação (CBIE 2016)

Anais do XXVII Simpósio Brasileiro de Informática na Educação (SBIE 2016)

Algumas telas não são interessantes, devido à falta de padrão - "Adotaria o padrão exibido em treinar. E melhoraria os ícones que indicam a letra inicial de cada palavra."; “... deixar a fonte mais bonita e menos formal, diferenciar os botões "Finalizar" e "Pausar", criar uma tela para o botão de "pause" e um menu".

Mais feedback para o usuário - "Penso que deveria ter alguma maneira de alertar que "bolhas" iriam aparecer e como jogar, pois demorei a perceber que devia escolher a palavra correta."; "Quando tocássemos na tela, escolhendo o grego, por exemplo, tivéssemos uma seção de curiosidades sobre aquela língua.".

Opção de configurar a aplicação - "O aplicativo não oferece opções de acessibilidade (aumentar/diminuir letras, leitura de textos, etc.) '”.

Durante essa análise, foi possível identificar também o nível de satisfação do participante, o item 5 na Figura 2, de acordo com cada aspecto avaliado no aplicativo. $\mathrm{Na}$ avaliação do aplicativo NaPontaDaLíngua, os participantes marcaram satisfeitos para oito dos 12 aspectos avaliados. Os participantes também marcaram nem satisfeitos nem insatisfeitos para três dos 12 aspectos avaliados. Já para o aspecto "Controle e liberdade do usuário", dez participantes marcaram insatisfeitos. Portanto, esse foi um dos pontos que gerou maior desagrado nos participantes, além de gerar sugestões de melhorias. Outro aspecto que também gerou desagrado e sugestões de melhorias foi o aspecto de "Consistência e padrões" para interface da aplicação.

Além disso, também foi pedida uma visão geral da satisfação com a aplicação. Os participantes avaliaram-na como satisfeitos. Essa análise do nível de satisfação é interessante para o pesquisador, pois torna possível identificar quais aspectos estão realmente satisfazendo o participante e qual os deixou insatisfeito [Nascimento et al. 2016].

Portanto, foi possível perceber como foi a experiência do usuário, através da análise de satisfação, detecção dos problemas e sugestão de melhorias, apontando quais pontos estão necessitando de melhorias e quais pontos estão satisfatórios para os participantes. Além disso, foi possível verificar que essas melhorias sugeridas estão relacionadas a experiências com aplicações utilizadas anteriormente, seja através de desejos ou necessidades relatadas pelo participante ao utilizar a aplicação. Essas melhorias sugeridas são úteis para aperfeiçoamento da aplicação, pois as mesmas poderiam melhorar a qualidade da aplicação, além de melhorar a experiência do usuário, a fim de gerar o sucesso na adoção e uso da aplicação [Charland e Leroux 2011].

Fazendo uma relação com possíveis impactos à aprendizagem, deve-se frisar que todas as melhorias sugeridas devem ser consideradas. Se um aplicativo demandar muito esforço para se realizar tarefas simples, isso pode gerar desânimo no aluno. Já erros e dificuldade de entendimento podem causar frustação e confusão no aprendiz. A falta de padronização das telas pode levar a uma sobrecarga cognitiva desnecessária. Sem feedback, o aluno pode se sentir perdido ou desamparado. E, por fim, todos os alunos devem ser contemplados e questões de acessibilidade devem sempre ser levadas em consideração. 
V Congresso Brasileiro de Informática na Educação (CBIE 2016)

Anais do XXVII Simpósio Brasileiro de Informática na Educação (SBIE 2016)

\section{Considerações finais}

Este artigo procurou apresentar o desenvolvimento de um aplicativo de apoio educacional elaborado no meio acadêmico. Antes de levá-lo às salas de aula, buscou-se avaliar sua Usabilidade e UX através de uma técnica também concebida no meio acadêmico e de outro método já amplamente utilizado. E que bom que isso foi feito! A grande quantidade de erros e defeitos encontrados causou espanto nos desenvolvedores do aplicativo.

No entanto, isso não desmotivou o trabalho, pois avaliações com esse tipo de resultado são semelhantes ao que a indústria adota e obtém. Pelo contrário, a pesquisa e o desenvolvimento em ambientes acadêmicos devem ser incentivados e aprimorados. Mas, conclui-se que é muito importante realizar a avaliação dos aplicativos e softwares e sistematizar alguns processos, antes de levá-los ao ambiente escolar. As sugestões de melhorias são ótimas fontes para a proposta de trabalhos futuros e versões mais robustas dos aplicativos e dos softwares.

\section{Agradecimentos}

Parte dos resultados apresentados neste artigo foi obtida através de atividades de P\&D do "Programa de Qualificação em Grande Escala em Tecnologias Móveis PROMOBILE", projeto patrocinado pela Samsung Eletrônica da Amazônia Ltda nos termos da lei federal brasileira ${ }^{0} 8.248 / 91$.

\section{Referências}

Atayde, A. P. R., Teixeira, A. B. M. e Pádua, C. I. P. S. (2003) "MAQSEI - Uma Metodologia de Avaliação de Qualidade de Software Educacional Infantil”, Simpósio Brasileiro de Informática na Educação (SBIE).

Bonifácio, B., Viana, D., Vieira, S., Araújo, C. e Conte, T. (2010) “Aplicando Técnicas de Inspeção de Usabilidade para Avaliar Aplicações Móveis”, IX Simpósio de Fatores Humanos em Sistemas Computacionais.

Catapan, A. H., Cornélio, F. P., Souza, A. D., Thomé, Z. and Cybys, W. D. A. (1999) "Ergonomia em software educacional: a possível integração entre usabilidade e aprendizagem", Workshop sobre fatores humanos em sistemas computacionais: rompendo barreiras entre pessoas e computadores. Campinas, São Paulo: UNICAMP/SEEC.

Charland, A. and Leroux, B. (2011). "Mobile application development: web vs. native", Communications of the ACM, v.54, n.5, 49-53.

De la Vara, J., Wnuk, K., Berntsson-Svensson, R., Sánchez, J. and Regnell, B. (2011) "An Empirical Study on the Importance of Quality Requirements in Industry," XXIII International Conference on Software Engineering and Knowledge Engineering SEKE 2011, Miami Beach, USA, 2011, pp. 438 - 443.

De Paula, D. F., Menezes, B. H. and Araújo, C. C. (2014) "Building a Quality Mobile Application: A User-Centered Study Focusing on Design Thinking, User Experience and Usability", Part of HCI International 2014. Design, User Experience, and 
V Congresso Brasileiro de Informática na Educação (CBIE 2016)

Anais do XXVII Simpósio Brasileiro de Informática na Educação (SBIE 2016)

Usability. User Experience Design for Diverse Interaction Platforms and Environments, v. 8518, p. 313-322.

Fernandez, A., Insfran, E. and Abrahão, S. (2011) "Usability evaluation methods for the web: A systematic mapping study", Information and Software Technology, v.53, n. 8, p. $789-817$.

Gaspar, W., Oliveira, E. e Oliveira, K. (2015). “Aprendizagem da Língua Portuguesa com Dispositivos Móveis: Um Mapeamento Sistemático da Literatura”, Simpósio Brasileiro de Informática na Educação, v.26, n.1, p. 140-149.

Gomes, A. S. e Padovani, S. (2005). "Usabilidade no ciclo de desenvolvimento de software educativo", Simpósio Brasileiro de Informática na Educação (SBIE).

International Organization for Standardization (2011) "Systems and software engineering - SQuaRE - Software product Quality Requirements and Evaluation System and software quality models", ISO/IEC 25010.

Kawakami, G., Rivero, L. e Conte, T. (2014) "Identificando Oportunidades de Melhoria em Tecnologias de Inspeção de Usabilidade para o Contexto Web Móvel através de um Experimento Controlado", In Proceedings of the XVII Ibero-American Conference on Software Engineering (CIBSE).

Matera, M., Costabile, M. F., Garzotto, F. and Paolini, P. (2002) "SUE Inspection: An Effective Method for Systematic Usability Evaluation of Hypermedia", In IEEE Transactions on Systems, Man and Cybernetics, Part A, v. 32, n. 1, p. 93-103.

Nascimento, I., Silva, W., Gadelha, B. and Conte, T. (2016). Userbility: A Technique for the Evaluation of User Experience and Usability on Mobile Applications. In International Conference on Human-Computer Interaction (pp. 372-383). Springer International Publishing.

Penedo, J. R., Diniz, M., Ferreira, S. B. L., Silveira, D. S. e Capra, E. (2012) “Análise de Usabilidade de um Sistema de EaD Baseada em Modelos Markovianos e em Taxonomia”, Simpósio Brasileiro de Informática na Educação (SBIE).

Reategui, E. (2007) "Interfaces para softwares educativos”, Revista Novas Tecnologias na Educação (Renote), 5(1).

Vermeeren, A., Law, E., Roto, V., Obrist, M., Hoonhout, J. and Väänänen-VainioMattila, K. (2010) "User Experience Evaluation Methods: Current State and Development Needs", In 6th Nordic Conference on Human-Computer Interaction: Extending Boundaries. ACM, p. 521-530. 